Background Growth in the first year of life may already be predictive of growth and obesity later in childhood. Early life exposure to endocrine disrupting chemicals (EDCs) has been associated with obesity in children and older populations.

Objective To assess the association between prenatal exposure to various EDCs and child growth in the first year of life.

Methods Cord plasma or breast milk was used to determine exposure to amongst others dichlorodiphenyldichloroethylene (DDE), mono (2-ethyl-5-oxohexyl) phthalate (MEOHP), and mono (2-ethyl-5-carboxypentyl) phthalate (MECPP). Data on weight and length until 11 months after birth was obtained. Mixed models were composed for each compound and health outcome. Exposure quartiles, time, and gender were added to the models as fixed effects. Subject was added as a random effect.

Results For MEOHP, boys in Q1 had a consistently higher BMI than higher exposed boys $(\mathrm{p}=0.029)$. MECPP exposure was related to increased BMI over time in both boys and girls in Q1, though the association was not significant $(\mathrm{p}=0.117)$. The effect of MECPP exposure on BMI was mainly due to weight, which was higher in the low exposed groups. For DDE interaction between time and exposure was significant $(\mathrm{p}=0.078)$. For boys in particular, those with relatively low exposures had higher BMI curves during the first year.

Conclusion Low exposure to phthalates and DDE was associated with BMI during the first year after birth. Results were gender specific, and associations were mostly non-monotonic. Follow-up is warranted to see if these effects are persistent during childhood.

\section{PS-358 EVALUATION OF PATIENTS WITH DIAGNOSIS OF FAMILIAL MEDITERRANEAN FEVER IN UMRANIYE REGION OF ISTANBUL}

N Basoglu, A Yazar, S Aydogdu, B Karakayali, S Akova, S Guven, I Islek. Pedatrics, Umraniye Training and Research Hospital, Istanbul, Turkey

\subsection{6/archdischild-2014-307384.657}

Background and aims A retrospective evaluation of clinical findings and genetic analysis of patients with Familial Mediterranean Fever (FMF) in Umraniye region of Istanbul.

Methods 44 patients with FMF were evaluated retrospectively in the Department of Paediatrics between 2013-2014 years.

Results The mean age of 44 patients was 8.8 years. Female-male ratio was 1.2:1. The clinical characteristics of patients were recorded as fever (84\%), abdominal pain (72.7\%), recurrent infection story (47.7\%), myalgia (43.1\%), arthritis $(15.9 \%$; monoarthritis, $11.4 \%$, polyarthritis $4.5 \%)$, pleuritis (11\%), erysipelas-like erythema (7\%), vasculitis (2.2\%). MEFV gene analysis in patients has revealed 8 mutations. The most common mutation type was M694V (47.7\%) followed by E148Q (38.6\%), R202Q (15.9\%), M680I (11.3\%), V726A (9\%), P369S (6.8\%), M694I and K695 (2.2\%), respectively. The M694V mutation was detected in 58\% of homozygous mutations. Patients with homozygous M694V mutation have shown significantly more often joint complaints when compared to patients with other mutations. The joint complaint was usually seen in the form of monoarthritis. The clinical immigrant polyarthritis ratio was $2.2 \%$.

Conclusions Although most previous genetic studies showed that M694V mutation was the leading locus of risk for developing amyloidosis, we couldn't determine amyloidosis in our study. In comparation with national studies, clinical signs of pleuritis, arthritis and erysipelas-like erythema determinated at lower rates in our study. The M680I mutation, which is very rare among Jews and relatively more prevalent in Aermenians and Arabs, was the fourth most common mutation in our study, although it was the second common mutation in nationwide studies.

\section{PS-359 CROSS-CULTURAL STUDY OF ADAPTATION IN FAMILIES OF INDIVIDUALS WITH DOWN SYNDROME}

M Van Riper, G Knafl. Nursing, University of North Carolina, Chapel Hill, USA

\subsection{6/archdischild-2014-307384.658}

Background There is growing evidence that while some families of individuals with Down Syndrome (DS) find it difficult to adapt to the ongoing challenges associated with raising an individual with DS, others adapt successfully and some even thrive. However, few studies have examined the experiences of families living in different countries. Therefore, the aim of this study was to examine the influence of family factors on adaptation in families of individuals with DS living in four countries.

Methods The guiding framework for this study was the Resiliency Model of Stress, Adjustment and Adaptation. Over 800 parents of individuals with DS from Ireland, Portugal, UK, and USA completed a survey which included these measures: Family Index of Regenerativity and Adaptation- General; Family Management Measure; Family Problem Solving Communication Index and the Brief Family Assessment Measure. Linear mixed modelling was used accounting for intra-familial correlation and constant variance for the two parents. An adaptive modelling process was also used.

Results Family functioning was worse with greater family strains and incendiary communication and with lower condition management ability, affirming communication, and family hardiness. Parent wellbeing was worse with greater condition management effort, family strains, family stressors and incendiary communication and with lower condition management ability and family hardiness.

Conclusion Findings contribute to our understanding of the underlying processes associated with differing outcomes in families of individuals with DS. Efforts to intervene will be more effective if clinicians recognise how culture and family factors interact and shape how families respond.

\section{PS-360 THE IMPACT OF CONGENITAL HEART DISEASE ON CHILDREN WITH DOWN SYNDROME ADMITTED FOR BRONCHIOLITIS}

${ }^{1} \mathrm{TW}$ Ting, ${ }^{2} \mathrm{PPC}$ Wong, ${ }^{3} \mathrm{D}$ Testoni, ${ }^{4} \mathrm{HH}$ Lee. ${ }^{1}$ Paediatric Medicine, KK Women's and Children's Hospital, Singapore, Singapore; 'Respiratory Medicine Service, KK Women's and Children's Hospital, Singapore, Singapore; ${ }^{3}$ Universidade Federal de Sao Paulo, Sao Paulo, Brazil; " Children's Intensive Care, KK Women's and Children's Hospital, Singapore, Singapore

\subsection{6/archdischild-2014-307384.659}

Background and aims Children with Down Syndrome (DS) are reported to have increased morbidity due to bronchiolitis. We aim to study if congenital heart defects will have an impact on children with DS admitted for bronchiolitis.

Methods We identified children with DS with diagnosis of bronchiolitis from 2004 to 2012 from electronic records. We define congenital heart defects (CHD) as all congenital heart defects except small patent ductus arteriosus or small atrial septal defect. 
Continuous and categorical data were summarised as median with interquartile ranges and proportions respectively. Continuous variables were compared with Mann Whitney rank-Sum test and categorical variables by Fisher exact test. Statistical significance was taken as $\mathrm{p}<0.05$ for all tests.

Results Twenty seven admissions were identified. The median age was 8 (5-18) months. Respiratory syncytial virus (37\%) was the most common virus isolated.

Fourteen (52\%) patients have CHD. There was no difference in proportion of children with DS and CHD requiring high dependency care (HD) compared to those without. (4/14 vs. 2/ $13, p=0.648)$. There was no difference in proportion of those with DS and CHD requiring intensive care (ICU) and those without $(1 / 14$ vs. $1 / 13, \mathrm{p}=0.999)$. There was no difference in median LOS between those with CHD and those without. (6.5 vs. 7 days, $\mathrm{p}=0.678)$. After excluding those CHD with corrective surgery done, there is still no significant difference between the two groups.

Conclusion We did not find any association between CHD and increase in morbidity among children with DS admitted for bronchiolitis.

\section{PS-361 EVALUATING PARENT SATISFACTION WITH MEDICAL CORRESPONDENCE FOLLOW UP METHODS AFTER A CARDIOLOGY OUTPATIENT CLINIC VISIT IN OUR LADY'S CHILDREN HOSPITAL, IRELAND}

${ }^{1} \mathrm{M}$ Bahari, ${ }^{2} \mathrm{~K}$ Walsh, ${ }^{3} \mathrm{D}$ McGill. 'Neonatology, National Maternity Hospital, Dublin, Ireland; ${ }^{2}$ Cardiology, Our Lady's Children Hospital, Dublin, Ireland; ${ }^{3}$ Department of Public Health and Policy, University of Liverpool, Liverpool, UK

\subsection{6/archdischild-2014-307384.660}

Background and aim Primary: To evaluate parents' satisfaction toward being copied into their GP letters after Paediatric Cardiology outpatient visits. Secondary: To explore acceptance of other methods of communication, and factors affecting acceptance.

Methods This was a quantitative, descriptive, cross sectional study assuming a positivistic approach. Questionnaires filled by participants were used to collect data in Cardiology Outpatient. September 10th to October 17th, 2009. Patients attending during the specified period were included; new referrals were excluded. A representative sample was calculated at 55 participants. 87 agreed to participate but only 66 returned questionnaire. Data was imported and analysed using PASW 18.

Results 97\% of Participants were satisfied when copied into GP letter. Parents discussion with a Paediatrician was the most helpful followed by GP, nurse, family member and other parents, with similar experience. Paediatrician was first choice for parents to discuss their children's health followed by GP, nurse, and other Parents. 60\% of Participants found the internet to be a helpful source for information, and 51\% found Patient Education Material (PED) helpful. Despite this Parents prefer PED to Internet. 94\% of Participants want to receive a copy of Paediatrician letter, but were divided over other methods of communication. Receiving a letter contributes significantly to parents' satisfaction $(\mathrm{p}=0.008)$. Gender and level of education was shown to influence participants' choice of communication.

Conclusion Parents hold high regards for being part of communication between health professionals. The use of medical terms does not render the usefulness of the letter to parents.

\section{PS-362 EPIDEMIOLOGY OF OROFACIAL CLEFTS IN EMILIA ROMAGNA AND TUSCANY REGIONS}

${ }^{1} \mathrm{M}$ Rubini, ${ }^{2} \mathrm{~S}$ Franchella, ${ }^{1} \mathrm{G}$ Astolfi, ${ }^{3} \mathrm{~F}$ Bianchi, ${ }^{1} \mathrm{P}$ Franceschelli, ${ }^{3} \mathrm{~A}$ Pierini, ${ }^{1} \mathrm{D}$ Balestra, ${ }^{4}$ A Franchella. ${ }^{1}$ Emilia Romagna Region, Imer, Ferrara, Italy; ${ }^{2}$ University Hospital, Otosurgery Unit, Padua, Italy; ${ }^{3}$ Tuscany Region, Malformations Register, Florence, Italy; ${ }^{4}$ Maternity and Childhood, Pediatric Surgery, Ferrara, Italy

\subsection{6/archdischild-2014-307384.661}

Background and aim Epidemiological information gathered through Birth defects surveillance is an important adjunct to carrying out clinical and etiological research.

Methods An Italian epidemiological investigation on Orofacialclefts (OFCs) conducted by the Congenital Malformation Registries of Emilia/Romagna (http://www.registroimer.it/) and Tuscany (http://www.rtdc.it/) in the period 2001-2011 identified 751 of OFC cases among 724.944 with an overall birth prevalence of 1.04/1,000. Birth prevalence of OFC variessignificantly in Europe ranging from 6,2 to 22,9 with a European mean value of1,45, showing a clear difference between the north and south of Europe (http://www.eurocat-network.eu/). The complex model of inheritance and the frequently conflicting results in different populations on the role of genes that constitute risk factors, suggest the presence of real biological differences.

Results Recorded cases included 166 (22\%) CL, 286 (38\%) CLP and 299 (40\%) CP. A predominance of males among CL (P) (M/ $\mathrm{F} 1,60)$ and of females among $\mathrm{CP}(\mathrm{M} / \mathrm{F} 0,79)$ as confirmed. Among 751 of OFC cases, 661 were live births (88.0\%), 7 stillbirths $(0.9 \%)$, while $83(11.1 \%)$ were terminations of pregnancy.522 cases (69\%) were isolated, 118 cases (16\%) OFC were present in recognised condition, and 111 cases $(15 \%)$ were associated with other congenital malformations (MCA). The study confirmed that cardiovascular (27\%), musculoskeletal (21\%) and central nervous system (21\%) defects are frequently associated.

Conclusions Thus a routine screening for other malformations may need to be considered in infants with OFC and a multidisciplinary approach of these patients to be organised starting from birth.

\section{PS-363 ACUTE PAEDIATRIC ADMISSION UNITS (APAU) CAN REDUCE OVERALL ADMISSIONS AND IMPROVE QUALITY OF CARE IN A DISTRICT GENERAL HOSPITAL (DGH) SETTING}

${ }^{1} \mathrm{G}$ McCall, ${ }^{2} \mathrm{NP}$ Corrigan. ${ }^{1}$ Paediatrics, Antrim Hospital, Belfast, UK; ${ }^{2}$ Paediatrics, Altnagelvin Hospital, Derry, UK

\subsection{6/archdischild-2014-307384.662}

Despite UK wide enthusiasm for Acute Paediatric Admission Units the wide variation in unit size and configuration makes generalisation of impact difficult. We believed that introducing an APAU in a DGH setting had the potential to reduce admissions, improve quality of patient care and help met $\mathrm{RCPCH}$ quality outcomes.

Aim To design, implement and audit an APAU with a defined patient pathway for all acute referrals. Primary outcomes would include admission rates, time to assessment, time to decision and questionnaires.

Method Retrospective data was collected to provide a baseline against which to judge efficacy of intervention. A designated area was identified and equipped as an APAU. Patient pathways were agreed along with a new admission proforma. A 4-week 